\title{
Cytologic Diagnosis of Bile Duct Strictures: Brush or Scrape?
}

\author{
Jason S. Gold ${ }^{1,2,3}$
}

Published online: 22 August 2018

(c) This is a U.S. Government work and not under copyright protection in the US; foreign copyright protection may apply 2018

Achieving a pathologic diagnosis for biliary strictures short of surgical resection is notoriously difficult, which in turn impairs the ability to deliver optimal clinical care. Confidence in classifying a biliary stricture as benign is limited since biopsies of strictures ultimately proven to be malignant yield the correct diagnosis only about half the time, an uncertainty which in turn leads to overtreatment. Approximately $10-15 \%$ of biliary strictures suspicious for malignancy are benign when analyzed following surgical resection, comprising a wide array of histopathologic entities $[1,2]$. As many medical oncologists are reluctant to systemically treat unresectable biliary strictures without a pathologic confirmation of malignancy, the inability to accurately diagnose these strictures also results in undertreatment. Furthermore, in the absence of a pathologic diagnosis, the treatment given may not always be appropriate since systemic treatment may be targeted against a suspected malignancy different from the one actually present (i.e., metastatic disease or hepatocellular carcinoma mimicking cholangiocarcinoma). Similarly, even when the correct histopathologic diagnosis is targeted, the inability to uniformly obtain adequate tissue limits the ability to provide personalized therapy.

For biliary strictures, tissue is often sampled via cholangiography (i.e., either endoscopic retrograde cholangiopancreatography [ERCP] or percutaneous transhepatic cholangiography [PTC]) in an attempt to achieve a diagnosis. Advantages of this strategy are that tissue sampling can be accomplished simultaneously with biliary drainage when needed, and in many cases, especially with extrahepatic cholangiocarcinomas, there is no obvious extraluminal mass

Jason S. Gold

jgold@bwh.harvard.edu

1 Surgical Service, VA Boston Healthcare System, 1400 VFW Parkway (112), West Roxbury, MA 02132, USA

2 Department of Surgery, Brigham and Women's Hospital, Boston, MA, USA

3 Harvard Medical School, Boston, MA, USA amenable to biopsy. In current practice, several techniques have been employed to obtain tissue for cytology or histopathology during cholangiography. The most commonly utilized approaches are bile aspiration, brush cytology and forceps biopsy, all of which have significant limitations. Sampling of bile for cytology, while quite simple, only has a reported sensitivity ranging from 6 to $32 \%$ [3-5]. The most commonly used method for tissue sampling during cholangiography, brush cytology, which is accomplished by placing specialized brushes into the bile duct, has a sensitivity of approximately $45 \%[6,7]$. The advent of forceps biopsy, in which tissue from a biliary stricture can be grasped and removed through the bile duct, was touted to improve the diagnostic yield over these other techniques. A meta-analysis comparing brush cytology to forceps biopsy did not reveal much improvement in the sensitivity for biliary forceps alone (48\%) over brush cytology, though the combination of brush cytology and forceps biopsy modestly but significantly improved sensitivity (59\%) beyond that of either technique used independently [7]. With any of these techniques, false-positive results are rare with specificities reported to be close to $100 \%$.

With the clear need for better methods to assess biliary strictures, scrape cytology, a novel technique in which a looped metallic wire is inserted into the bile duct during ERCP so as to scrape the stricture in order to obtain a cytologic specimen, was recently developed and first applied for the diagnosis of pancreatic ductal strictures [8]. Recently, a prospective multicenter trial comparing forceps biopsy with scrape cytology was reported [9] in which ERCP was attempted in 123 patients in whom a malignant biliary stricture was suspected. A guidewire could be passed into the bile duct in $97 \%$ of cases; in these, forceps biopsy was first attempted followed by biliary scraping. In cases where the bile duct was cannulated, the forceps biopsy was able to reach the stricture $90 \%$ of the time and adequate tissue was obtained in $86 \%$. In comparison, the biliary scraper could reach the stricture in $99 \%$ of cases and obtained adequate tissue in $97 \%$. In the cases with malignant strictures, cancer was detected in $51 \%$ with biopsy forceps, $65 \%$ with scrape 
cytology and $75 \%$ with the combination. Both the biliary scraper and the combination were statistically superior to forceps biopsy alone. This study did not exclude from analysis cases where the bile duct could not be cannulated or where sufficient tissue could not be obtained, so while the manuscript gives a good 'real-world' estimate of the utility of these techniques, it does not answer the question of how much the observed superiority of scrape cytology was driven by its superior ability to obtain tissue as opposed to its ability to make a diagnosis based on the tissue obtained. Also, it is not known if the yield of scrape cytology was improved by performing forceps biopsy first.

In light of these problems, an article published in this issue of Digestive Diseases and Sciences by Nakahara et al. from St. Marianna University School of Medicine in Japan offers a retrospective, single-center experience in which the authors compare the results of specimens obtained with scrape cytology in 79 patients to those with obtained conventional cytology in a historical control group of 341 patients [10]. Nakahara et al. are to be commended for attempting to overcome some of the limitations of the previous trial and to extend the experience with scrape cytology via ERCP for the diagnosis of biliary strictures. The sensitivity of scrape cytology (41\%) was significantly greater than that of conventional cytology (27\%). As there was heterogeneity in the case mix between the two groups, and as this study and other studies have noted superior sensitivity of cytology specimens obtained via cholangiography for the diagnosis of primary biliary malignancies as opposed to other tumors, the authors analyzed their results separately for pancreas cancer and for biliary cancer. The sensitivity of scrape cytology for biliary cancer was $52 \%$ compared to $38 \%$ for conventional cytology, which approached but did not achieve significance $(p=0.10)$. Since forceps biopsy was performed in nearly all of the patients who had scrape cytology and in approximately half of the patients who had conventional cytology, the sensitivity of conventional cytology and scrape cytology was compared to forceps biopsy. Forceps biopsy was more sensitive than conventional cytology (36-27\%) but had a similar sensitivity to scrape cytology $(40-41 \%)$. Again, since the case mix could impact these results, the data were analyzed for pancreas cancer and biliary cancer separately. Forceps biopsy was $50 \%$ sensitive for biliary cancer compared with $38 \%$ for conventional cytology, a result that approached statistical significance $(p=0.08)$, whereas in the scrape cytology group, forceps biopsy had an equivalent sensitivity for biliary cancer as compared to scrape cytology (55 vs. $52 \%$ ).

This study was limited by the heterogeneity of the case mix and the relatively small numbers of patients with each diagnosis. Thus, while the results suggest that scrape cytology is superior to conventional cytology for the diagnosis of biliary cancers, statistical significance was not reached for these important comparisons. The direct comparison of the scrape cytology group to the conventional cytology group is difficult to interpret given the variation in the incidence of the different diseases between the groups and the marked differences in the efficacy of these techniques depending on the disease process. Another important limitation is that aspiration cytology was performed in the vast majority of cases in the conventional cytology group (312), whereas brush cytology was only performed in a small number (29). As brush cytology is the more commonly performed technique in US clinical practice and has a higher reported sensitivity, a direct comparison between scrape cytology and brush cytology is still needed. Moreover, since the cytology results were all processed and interpreted at the time of the initial procedure, it is not known whether any lower sensitivity in the historical group may be attributable to improvements in cytopathologic interpretation over the time period of the study.

The results achieved in this study with scrape cytology (i.e., the sensitivity of 52\% for biliary cancer and 30\% for pancreas cancer) are not as favorable as those achieved in the previously mentioned multicenter trial $(74 \%$ and $42 \%$, respectively). It is possible that this may be due to the fact that forceps biopsy was performed first in the multicenter trial, potentially better exposing or disrupting the lesion for retrieval by scrape, whereas scrape biopsy was performed before forceps biopsy in this study.

Overall, in combination with the previous multicenter trial, this new study furthers the published experience with this new technique. While this publication does not definitively find any evidence of superiority of scrape biopsy over conventional cytology for the diagnosis of biliary strictures, it provides, along with the prior trial, ample rationale for continued investigation. In addition to assessing the safety and efficacy of biliary scrape cytology, the results for this technique will need to be compared to other techniques such as endoscopic ultrasound-guided fine-needle aspiration (EUS FNA), which may be superior to brush cytology and forceps biopsy [11], and will need to be evaluated in the context of advanced techniques for interpreting cytologic specimens including digital image analysis (DIA) and fluorescence in situ hybridization (FISH). In all, scrape cytology represents a potentially important advance in the diagnosis of suspected malignant biliary strictures using minimally invasive methods.

\section{Compliance with ethical standards}

Conflict of interest The author declares that they have no conflict of interest. 


\section{References}

1. Corvera CU, Blumgart LH, Darvishian F, et al. Clinical and pathologic features of proximal biliary strictures masquerading as hilar cholangiocarcinoma. J Am Coll Surg. 2005;201:862-869.

2. Scheuermann U, Widyaningsih R, Hoppe-Lotichius M, Heise M, Otto G. Detection of benign hilar bile duct stenoses-a retrospective analysis in 250 patients with suspicion of Klatskin tumour. Ann Med Surg (Lond). 2016;8:43-49.

3. Kurzawinski TR, Deery A, Dooley JS, Dick R, Hobbs KE, Davidson BR. A prospective study of biliary cytology in 100 patients with bile duct strictures. Hepatology. 1993;18:1399-1403.

4. Fogel EL, Sherman S. How to improve the accuracy of diagnosis of malignant biliary strictures. Endoscopy. 1999;31:758-760.

5. Yamaguchi T, Shirai Y, Nakamura N, et al. Usefulness of brush cytology combined with pancreatic juice cytology in the diagnosis of pancreatic cancer: significance of pancreatic juice cytology after brushing. Pancreas. 2012;41:1225-1229.

6. Burnett AS, Calvert TJ, Chokshi RJ. Sensitivity of endoscopic retrograde cholangiopancreatography standard cytology: 10-y review of the literature. J Surg Res. 2013;184:304-311.
7. Navaneethan U, Njei B, Lourdusamy V, Konjeti R, Vargo JJ, Parsi MA. Comparative effectiveness of biliary brush cytology and intraductal biopsy for detection of malignant biliary strictures: a systematic review and meta-analysis. Gastrointest Endosc. 2015;81:168-176.

8. Uehara H, Tatsumi K, Masuda E, et al. Scraping cytology with a guidewire for pancreatic-ductal strictures. Gastrointest Endosc. 2009; 70:52-59.

9. Sakuma Y, Kodama Y, Sogabe Y, et al. Diagnostic performance of a new endoscopic scraper for malignant biliary strictures: a multicenter prospective study. Gastrointest Endosc. 2017;85:371-379.

10. Nakahara $\mathrm{K}$, Michikawa $\mathrm{Y}$, Morita $\mathrm{R}$ et al. Diagnostic ability of endoscopic bile cytology using a newly designed biliary scraper for biliary strictures. Dig Dis Sci. (Epub ahead of print). https:// doi.org/10.1007/s10620-018-5217-y.

11. De Moura DTH, Moura EGH, Bernardo WM, et al. Endoscopic retrograde cholangiopancreatography versus endoscopic ultrasound for tissue diagnosis of malignant biliary stricture: systematic review and meta-analysis. Endosc Ultrasound. 2018;7:10-19. 